$$
t=-\frac{P_{1}-p_{1}}{\partial p_{i} / \partial t-\partial p_{w} / \partial t}
$$

Now we apply Raoult's law and, accordingly, substitute for $P_{1}-p_{1}$ its equal $P_{1}(1-x)$, as in section 3 . Remembering further, that $x$, the molar fraction of the solvent, is

$$
x=\frac{\mathrm{I} \infty \mathrm{O} / M_{0}}{\mathrm{I00} / M_{0}+\Delta / M^{\prime}}
$$

and that the solution is infinitely dilute, equation (5) becomes:

$$
t=-\frac{P_{1} \Delta / M}{\left(\mathrm{I} 00 / M_{0}\right)\left(\partial p_{i} / \partial t-\partial p_{w} / \partial t\right)}
$$

Finally, since $M_{0}=R T / P_{1} v$, where $v$ is the specific volume of the vapor of the solvent, the depression $E$ per one mol of solute becomes:

$$
E=-\frac{R T}{100 v\left(\partial p_{i} / \partial t-\partial p_{w} / \partial t\right)}
$$

Unlike the corresponding expression for the molecular elevation of the boiling point, this expression (6) for the constant of cryoscopy has scarcely more than theoretical interest; for the difference of the two slopes involved is usually small and difficult to determine with precision. Here van't Hoff's formula, based on the heat of fusion of the solvent, will usually be of greater practical advantage. Both expressions (2) and (6) turn into the commonly used Van't Hoff formulae if combined with the ClapeyronClausius equation; for ebullioscopic purposes, as we have seen, this transformation is not desirable.

In conclusion we would point out that, since Raoult's law follows from the Duhem-Margules equation, and since the molar fractions in the latter are based on the molecular weights, not in the liquid mixture, but in the vapors emitted by it - the curious idea suggests itself that all ebullioscopic and cryoscopic measurements indicate molecular weights, not really of the substances in solution, but of their vapors emitted by the solution. That the molecular weight of the "solvent" in Raoult's law is that of the solvent's vapor, is generally recognized.

Worcester, Mass.

[Contribution from the Chemical laboratories of NeW Hampshire College.]

\title{
THE SEPARATION OF YTTRIUM FROM THE YTTRIUM EARTHS. PART II.
}

By H. C. Holden and C. James.

Received May 13, 1914.

In a previous paper, the authors have shown several methods of separating yttrium from the yttrium earths, the most efficient of which were, the fractional precipitation of the chromates and the fractional precipi- 
tation of the phosphates. These methods, however, were not well adapted to the separation of yttrium on a commercial scale, and therefore the work was continued in hope of finding a suitable method to use in working up large quantities of material.

\section{A. Fractional Precipitation by Means of Sodium Nitrite.}

The material first used was the most soluble portion obtained during the fractionation of the yttrium earths from Carolina monazite by the bromate method. It contained ytterbium, lutecium, erbium, thulium and yttrium with traces of thorium. A quantity of this material was converted into the oxide dissolved in nitric acid and diluted to about $1500 \mathrm{cc}$. with water. This was boiled and stirred by means of steam, while an amount of a concentrated solution of sodium nitrite was added sufficient to precipitate about one-third the rare earth material present. In a like manner, another precipitate was obtained from the mother liquor. Nitric acid was then added to the filtrate from Fraction 2 in order to liberate the nitrous acid and Fraction 3 was obtained by means of oxalic acid. These fractions were then purified by reprecipitating as oxalates, igniting, boiling the oxides with water to free them from sodium, dissolving in hydrochloric acid, reprecipitating as oxalate and igniting. A portion of each was then taken and the equivalent determined by the method described in Part I.

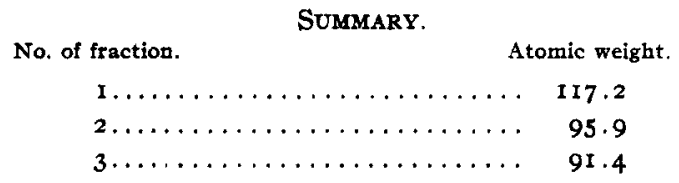

Fraction I was then dissolved in nitric acid and diluted to about 750 cc. with water, a small quantity of sodium nitrite was added and the solution placed in a flask, where the pressure was reduced considerably at room temperature. In this manner Fraction IA was obtained and proved to be thorium. The mother liquor was then fractionated further by means of sodium nitrite. The method was similar to that used in the first case, except that a smaller quantity of sodium nitrite was added each time. Five fractions were thus obtained, and, after purification, the equivalents were determined.

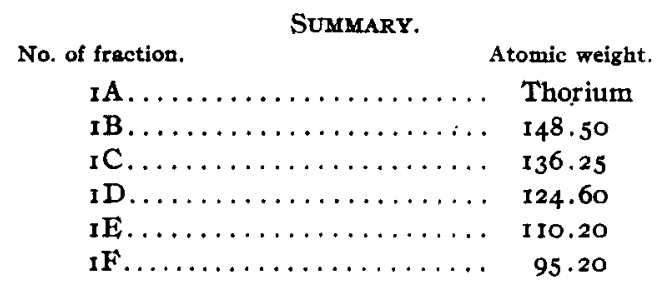

Fraction IB was nearly rose color and gave a fairly dense oxalate. Frac- 
tion $\mathrm{IC}$ was a light pink color and gave an oxalate less dense than $\mathrm{IB}$. $\mathrm{ID}$ was more pink than $\mathrm{IC}$ and also denser. $\mathrm{IE}$ was lighter in color than I $D$ but was about the same density. Fraction $\mathrm{IF}$ was lighter colored than $\mathrm{IE}$ and gave a very fluffy oxalate.

Fraction 2 was then dissolved in nitric acid and diluted to about $700 \mathrm{cc}$. with water, boiled and stirred as before. By adding a sufficient amount of sodium nitrite to precipitate about one-fourth the rare earth material present, Fraction $2 \mathrm{~A}$ was obtained. In the same way, three more fractions were obtained and then nitric acid was added to free the solution of nitrous acid, after which oxalic acid was added to get Fraction $2 \mathrm{E}$. These were then purified and the equivalents determined.

SUMmary.

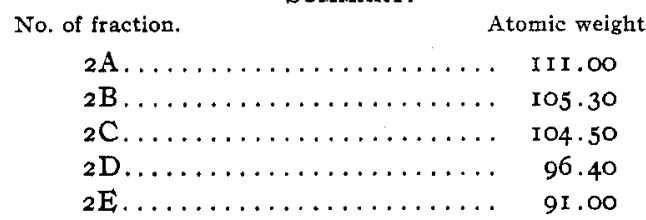

Fraction 2A was pink and fairly dense, the color of the series gradually grew lighter to $2 \mathrm{E}$, which was just slightly pink. The poor separation between $2 \mathrm{~B}$ and $2 \mathrm{C}$ was due to the fact that the precipitate was colloidal and not granular as the others.

Fraction 3 was next dissolved in nitric acid, diluted to about $700 \mathrm{cc}$. with water and four fractions obtained in a similar manner to that in the previous case. These were purified as before and the equivalents determined.

\begin{tabular}{|c|c|}
\hline \multicolumn{2}{|c|}{ SUMMARY. } \\
\hline No. of fraction. & Atomic weight. \\
\hline${ }_{3} \mathrm{~A} \ldots$ & 94.00 \\
\hline $3 \mathrm{~B}$. & 91.10 \\
\hline${ }_{3} \mathrm{C}$. & 89.50 \\
\hline${ }_{3} \mathrm{D} \ldots$ & $\cdots$ \\
\hline
\end{tabular}

Fraction $3 \mathrm{~A}$ was similar in color to Fraction $2 \mathrm{C}$ and was fairly dense. ${ }_{3} \mathrm{~B}$ was nearly white with about the same density as $3 \mathrm{~A}$. ${ }_{3} \mathrm{C}$ was white with a tinge of yellow. $3 \mathrm{D}$ had a tendency toward pale buff and there was not a sufficient quantity to make an equivalent determination. Fractions $3 \mathrm{~A}, 3 \mathrm{~B}$, and ${ }_{3} \mathrm{C}$ were about of equal size. By means of the spectrograph it was found that IB gave an intense ytterbium spectrum while ID contained no ytterbium. Fraction $3 \mathrm{C}$ consisted of practically pure yttrium. This can be seen to be the most efficient and rapid method of separation found up to the present time.

Since the separation of yttrium from dysprosium and holmium is more difficult than from erbium, it was thought that it would be of interest to apply the nitrite method to a mixture of yttrium, dysprosium, and holmium, 
containing traces of terbium and neodymium. About $50 \mathrm{~g}$. of these oxides were dissolved in nitric acid and diluted to $800 \mathrm{cc}$. with water. The method of fractionation was the same as in the previous case, except that a large excess of sodium nitrite was added at the very beginning of the fractionation. The first three fractions obtained were very markedly colloidal and could not be filtered, but had to be allowed to stand over night to settle. The final fraction was taken by means of oxalic acid after freeing from the nitrous acid. The fractions were then purified and the equivalents determined.

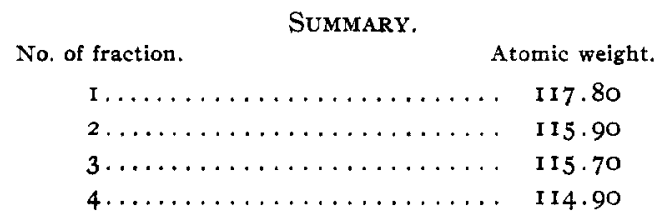

The fact that the separation was very inefficient was probably due to the colloidal condition of the precipitate obtained. After a careful investigation of the variables involved in the fractionation, it was found that a very large excess of sodium nitrite would cause the precipitate to be colloidal instead of granular. Therefore, the same material was taken and subjected to fractionation again, care being taken to avoid an excess of sodium nitrite. Eight fractions were thus obtained, the last one being precipitated with oxalic acid. It took considerable boiling to obtain the fractions and the precipitate was very fine and fil tered readily. Terbium was found to collect in the first fractions, while neodymium was concentrated in the last fraction and was removed by means of sodium sulfate. These various fractions were then purified as before and their equivalents determined.

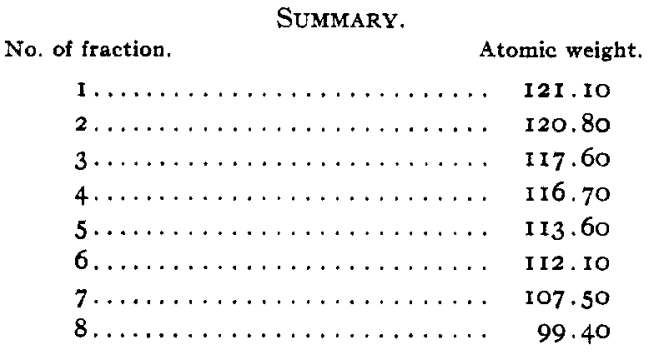

A mixture of the oxides of yttrium and erbium was dissolved in nitric acid, diluted to about one liter and subjected to the same method of fractionation. In the first case, five fractions were obtained by using a large excess of nitrite. The somewhat colloidal precipitates were purified as before and as there was no apparent separation when examined by the spectroscope, the equivalents were not determined.

The same material was taken and fractionated again, with only.a slight 
excess of sodium nitrite over the amount required to precipitate the first fraction. Six fractions were obtained, all of which were crystalline and filtered rapidly. These were purified and their equivalents determined as before. A gradual change of color could be seen in going from Fraction 1, which was pink, to Fraction 6, which was pure white.

SUMMary.

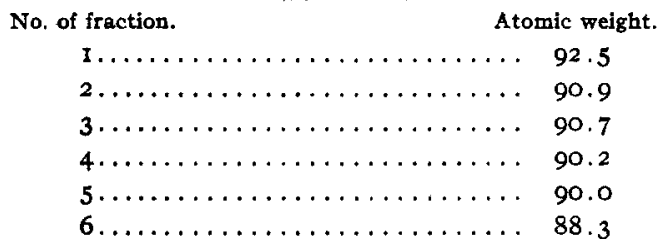

By this method about $20 \%$ of the original material was obtained in the last fraction, which was pure yttrium.

Fractionation of Gadolinite Material. - About $300 \mathrm{~g}$. of rare earth oxides, obtained from gadolinite, were dissolved in nitric acid and diluted to about three liters with water. This was boiled in an enamel pail and sodium nitrite added in small amounts until a fair sized precipitate was formed. In this way eight fractions were obtained. The first and second precipitates were somewhat gelatinous, but the succeeding ones grew more granular and filtered readily. Upon examination, the first fraction was found to contain thorium. Fraction 2 showed a strong spectrum of erbium. Fraction 3 gave a strong erbium spectrum and a trace of neodymium. Fraction 4 showed a decrease in the erbium spectrum and a trace of neodymium. Fraction 5 gave a further decrease in the erbium with an increase in the neodymium. Fraction 6 showed an increase in the neodymium and a trace of erbium. In Fraction 7 a still further increase in the neodymium and a trace of erbium were apparent. Fraction 8 gave an increase in the neodymium and no erbium whatsoever. The last three fractions contained about one-fifth the original material. From these results it would seem that the yttrium could be separated from the gadolinite earths very rapidly.

\section{B. Fusion of the Nitrates.}

A mixture of erbium and yttrium oxides were dissolved in nitric acid and the resulting nitrates evaporated and fused until brown fumes began to be evolved. This fused mass was then poured into a casserole containing cold water and then dissolved by heating and evaporated just a slight amount. The basic nitrates were then allowed to crystallize out by standing over night. The crystals thus obtained formed Fraction I and the filtrate was submitted to the same treatment as before to obtain Fraction 2. In this way six fractions were obtained. Fraction 7 was precipitated from the filtrate of Fraction 6 by means of oxalic acid and contained about one-fourth the original material. 
SUMMARY.

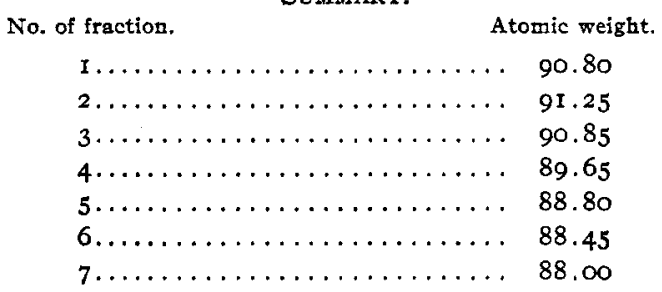

As can be seen, Fraction I gave an atomic weight less than Fraction 2. This was due to the fact that the decomposition was carried too far and therefore the basic nitrate obtained was somewhat colloidal and also the fused nitrate was not entirely soluble in water.

\title{
C. Fractionation by Means of Boiling with Sodium Hydroxide.
}

A concentrated nitrate solution containing yttrium and erbium was boiled, and a sufficient quantity of sodium hydroxide added to precipitate about one-fifth the rare earth material present. This was boiled for a short time and then allowed to stand over night in order that the basic nitrate might crystallize out. In this way three fractions were obtained and the fourth precipitated with oxalic acid. These were purified as before and the equivalents determined.

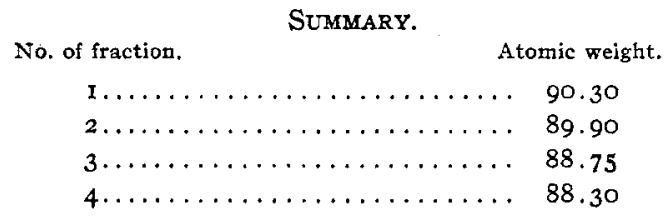

Conclusions.

The best method found thus far for separating yttrium efficiently from the other earths is by means of fractional precipitation with sodium nitrite. This gives a larger yield, a more rapid separation and is less expensive than either the phosphate or chromate method. This method is, however, not very effective for separating terbium from yttrium.

\section{CONTRIBUTION TO THE CHEMISTRY OF GOLD. II. AUTO- REDUCTION AS A FACTOR IN THE PRECIPI- TATION OF METALLIC GOLD.}

\author{
BY VICTOR IENHER. \\ Received April 27, 1914.
}

In the various studies which have been made on the precipitation of gold from solution, attention has been directed for the most part to the action of various reducing agents on gold solutions. The general chemical inactivity of gold enables us to deposit the metal in elementary form by most of the metals, the metallic sulfides, ferrous salts, various organic com- 